\title{
Critical causes in severe bleeding requiring angioembolization after percutaneous nephrolithotomy
}

\author{
Hee Youn Kim¹', Kyu Won Lee ${ }^{2}$ and Dong Sup Lee ${ }^{1 *}$
}

\begin{abstract}
Background: To identify the risk factors for severe bleeding requiring angioembolization among patients who received transfusions after $\mathrm{PCNL}$, particularly those who underwent anatomically incorrect renal puncture.

Methods: A total of 53 patients, who received transfusions after PCNL and simultaneously had a postoperative CT scan performed between November 2009 and May 2019 at two teaching hospitals, were retrospectively reviewed. The patients were divided into two groups: those who underwent angioembolization and those who did not. Patient, stone and procedural factors were compared between the two groups. Puncture correctness was evaluated using postoperative CT scans. Puncture was defined as being a correct puncture if the fornix or papilla of the posterior calyx was punctured and the trajectory of the tract was within 20 degrees posterior to the frontal plane of the kidney (i.e., within Brödel's line).

Results: 21 patients underwent angioembolization after PCNL. Incorrect puncture was seen in 14/21 (66.7\%) patients who underwent angioembolization after PCNL, whereas it was seen in 11/32 (34.4\%) patients who did not undergo angioembolization $(p=0.021)$. On multivariable regression analysis, puncture correctness was found to be the only significant factor, with an OR of $3.818,95 \% \mathrm{Cl}$ of $1.192-12.231$ and $p$ value of 0.024 .

Conclusions: Incorrect renal puncture was related to severe bleeding requiring angioembolization after PCNL. Our results emphasize the importance of the basic principle of renal puncture for PCNL.
\end{abstract}

Keywords: Kidney calculi, Percutaneous nephrolithotomy, Hemorrhage, Therapeutic embolization

\section{Background}

Anatomical understanding of the pelvocalyceal system and the related renal vasculature is essential to prevent bleeding complications during percutaneous nephrolithotomy (PCNL). Brödel's line of bloodless incision, the relatively avascular plane where the anterior and posterior segmental renal artery branches meet, is the key anatomical area [1]. Historically, before the era of PCNL, when anatrophic nephrolithotomy was performed,

\footnotetext{
* Correspondence: bbangbbangi@naver.com

'Department of Urology, St. Vincent's Hospital, College of Medicine, The Catholic University of Korea, 93, Jungbu-daero, Paldal-gu, Suwon-si, Gyeonggi-do 16247, Republic of Korea

Full list of author information is available at the end of the article
}

incision of the kidney was performed through this Brödel's line to minimize bleeding. The same principle applies to the PCNL procedure. The puncture should ideally traverse the relatively avascular Brödel's line, thereby decreasing the risk of bleeding. This is why the posterior calyx is considered the optimal calyx to puncture because posterior calices are usually oriented towards Brödel's line [2-4].

When the puncture is made outside of Brödel's line, the risk of arterial injuries increases, potentially leading to persistent and severe bleeding. Although rare, injury to these arteries can cause hemodynamic instability and can be potentially life-threatening. A high degree of 
suspicion, prompt angiography and subsequent angioembolization are required in these instances.

Past reports that studied risk factors for angioembolization after PCNL did not consider whether anatomically correct puncture was performed $[5,6]$. In the current study, we aimed to identify risk factors of severe bleeding requiring angioembolization among patients who received transfusions after PCNL, particularly those involved in anatomically correct renal puncture (i.e., through Brödel's line).

\section{Methods}

\section{Study population and design}

The current study used a retrospective design. A chart review of all patients who received transfusions after PCNL between November 2009 and May 2019 at two teaching hospitals was performed. Among these patients, only those who had undergone a postoperative computer tomography (CT) scan to evaluate the cause of bleeding were included. Patients with kidney anomalies (e.g., horseshoe kidney, calyceal diverticulum, etc.), and patients who underwent simultaneous bilateral procedures were excluded. The included patients were divided into two groups: patients who did not undergo angioembolization (No AE) and patients who underwent angioembolization (AE). Patient, stone and procedure-related characteristics were recorded and compared between the two groups. Patient characteristics included age, sex, body mass index (BMI) and American Society of Anesthesiologists (ASA) classification. Stone characteristics included laterality, stone location, stone size, staghorn stone, Guy's stone score [7], Hounsfield unit, degree of hydronephrosis, and preoperative percutaneous nephrostomy. The degree of hydronephrosis was evaluated using the criteria of the Society for Fetal Urology (SFU) [8]. SFU grade 0 was defined as 'no hydronephrosis', SFU grades I-III was defined as 'mild hydronephrosis', and SFU grade IV was defined as 'severe hydronephrosis'. Procedure-related characteristics included operation time, location of access (lower pole, other (middle or upper), and lower pole plus other), number of tracts, puncture correctness and stone free rate. Puncture correctness was evaluated using postoperative CT scans. Puncture was deemed a correct puncture if the fornix or papilla of the posterior calyx was punctured and the trajectory of the tract was within 20 degrees posterior to the frontal plane of the kidney (i.e., within Brödel's line) (Figs. 1 and 2) [2-4]. Stone free rate was evaluated with postoperative CT scans within months. Residual fragments under $2 \mathrm{~mm}$ was considered insignificant. Details of the angioembolization were also reviewed. The interval between the surgery and angioembolization, and the angiographic findings were recorded.

\section{Surgical technique}

Here, the PCNL procedure is briefly described. A ureteral occlusion balloon catheter was inserted via a cystoscope. All PCNL procedures were performed in the prone position with fluoroscopic assistance. A balloon dilatator (X-FORCE ${ }^{\circledR}$ N30 Nephrostomy Balloon Dilation Catheter, Bard Medical, GA, USA) was used for tract dilatation, and a 30F Amplatz sheath was used. A rigid 24F nephroscope (Richard Wolf, Knittlingen, Germany) was used for stone fragmentation and extraction. An antegrade ureteral catheter and a $20 \mathrm{~F}$ nephrostomy tube were placed at the end of the operation.

\section{Data analysis}

SPSS (IBM Corp. Released 2012. IBM SPSS Statistics for Windows, Version 21.0. Armonk, NY: IBM Corp.) was used for the statistical analysis. The comparison of

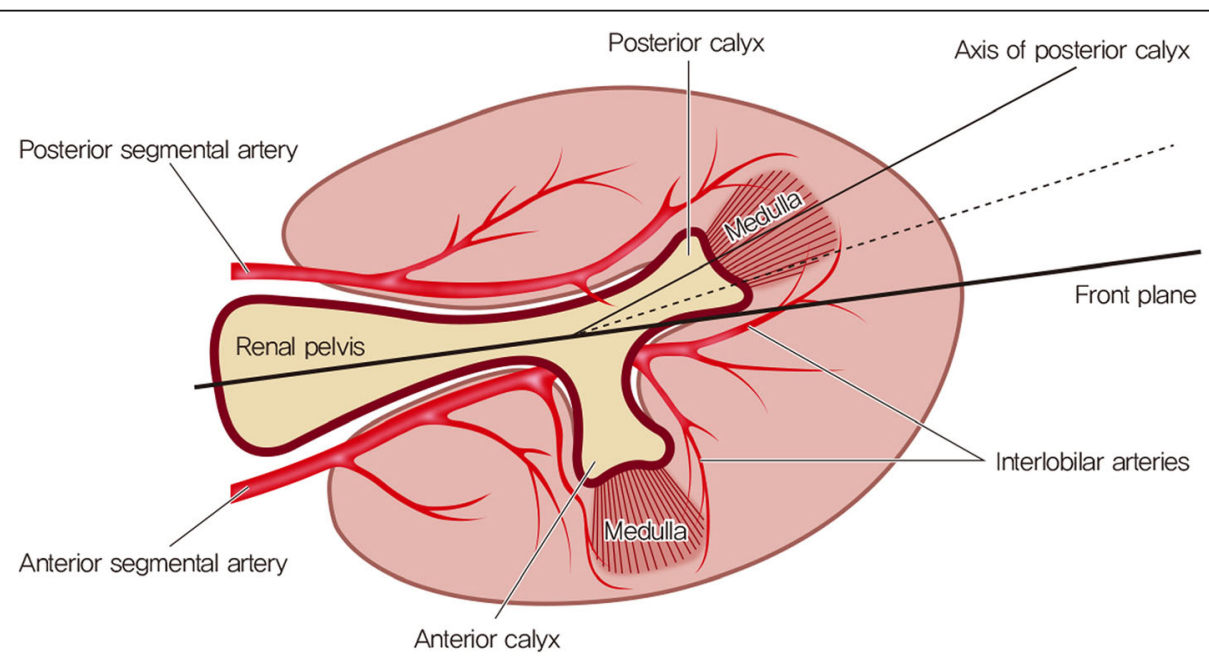

Fig. 1 Anatomy of axial view of kidney. Puncture was defined as being a correct puncture if the fornix or papilla of the posterior calyx was punctured and the trajectory of the tract was within 20 degrees posterior to the frontal plane of the kidney (i.e., within Brödel's line) 


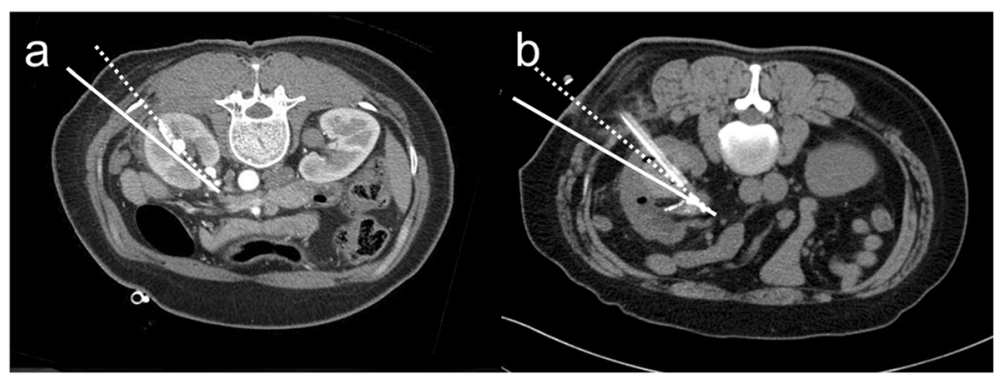

Fig. 2 Example of a CT scan of a correct (a) and incorrect (b) puncture. Solid line represents the frontal plane, and dotted line represents the line 20 degrees posterior to the frontal plane. In the CT scan of correct puncture (a), the tract is punctured within Brödel's line. In the CT scan of incorrect puncture (b), the tract is punctured outside of Brödel's line

continuous variables was performed using the unpaired $\mathrm{t}$-test or the Mann-Whitney test based on the result of the Shapiro-Wilk test for normality. The comparison of categorical variables was performed using the chi-square test or Fisher's exact test. A multivariate logistic regression analysis was conducted to investigate the risk factors of angioembolization. A $p$ value $<0.05$ was considered significant.

\section{Results}

The baseline patient and stone characteristics of the two groups are described in Table 1 . Of the 1554 patients who underwent PCNL during the study period, $53 \mathrm{pa-}$ tients received blood transfusion after the surgery and simultaneously underwent a postoperative CT scan. 32 patients did not undergo angioembolization (No $\mathrm{AE}$ group) and 21 underwent angioembolization (AE group). The rate of angioembolization after PCNL was 1.4\% (21/ 1554). There was no difference in age, BMI or ASA classification between the two groups. A statistically significant male predominance was noted in the AE group ( $43.8 \%$ versus $71.4 \%, p=0.048)$. There was no difference in stone laterality, stone location, stone size, presence of staghorn stone, Guy's stone score, Hounsfield unit, degree of hydronephrosis, or preoperative PCN.

Procedure-related characteristics are described in Table 2. There was no difference in operation time, location of access, number of tracts and stone free rate between the two groups. Puncture correctness showed a statistically significant difference, where correct puncture was noted in $65.6 \%$ of the No AE group and $33.3 \%$ of the $\mathrm{AE}$ group $(p=0.021)$. Incorrect puncture in patients who underwent angioembolization occurred approximately twice as often as in patients who did not undergo angioembolization.

Multivariable logistic regression analysis was performed to identify predictive factors of angioembolization after percutaneous nephrolithotomy and the results are shown in Table 3. Puncture correctness was the only factor that showed statistical significance, with an odds ratio (OR) of $3.818,95 \%$ confidence interval (CI) of $1.192-12.231$ and $p$ value of 0.024 .

\section{Discussion}

Despite the increasing transition to a less invasive surgical method for removal of kidney stones such as retrograde intrarenal surgery in recent years, percutaneous nephrolithotomy (PCNL) is still an integral part of treatment for large kidney stones [9]. Through decades of surgical experience with PCNL since it gained popularity after Fernström and Johannson's report in 1976 [10], PCNL has proven to be an effective surgery for kidney stone removal.

Nevertheless, the reported complication rate of PCNL, although lower than the open approach, is relatively high. Bleeding is the most common and significant complication of PCNL, with the reported incidence of bleeding requiring transfusion as high as $20 \%$, with $7 \%$ as the average incidence [11]. Most bleeding complications result from venous injuries, which improve with conservative care such as transfusion and usually do not cause hemodynamic instability. However, when the cause of bleeding is arterial injuries, persistent and severe bleeding may occur that does not improve with transfusion alone, leading to hemodynamic instability. Prompt angiography and angioembolization are needed in these cases. Although the reported incidence of angioembolization after PCNL is not high, ranging between 0 and $1.5 \%$ [11], it can potentially be life-threatening if prompt measures are not undertaken. This retrospective study was conducted to determine what separates patients who suffer severe bleeding requiring angioembolization after PCNL from patients who bleed enough to receive transfusion but recover without further measures.

Several past studies have attempted to identify factors involved in angioembolization after PCNL. Srivastava et al. retrospectively analyzed 1854 patients and identified 27 (1.4\%) patients who required angiography [6]. In the multivariate analysis, stone size was the only 
Table 1 Comparison of baseline information between patients who did not undergo angioembolization and patients who underwent angioembolization after percutaneous nephrolithotomy

\begin{tabular}{llll}
\hline & No AE & AE & $p$ value \\
\hline $\begin{array}{l}\text { Number of cases } \\
\text { Sex }\end{array}$ & 32 & 21 & \\
$\quad$ Male & $14 / 32(43.8 \%)$ & $15 / 21(71.4 \%)$ & \\
$\quad$ Female & $18 / 32(56.3 \%)$ & $6 / 21(28.6 \%)$ & \\
Age (years) & $58.9 \pm 12.2$ & $61.3 \pm 13.9$ & $0.511^{\text {b }}$ \\
BMI (kg/m $\left.{ }^{2}\right)$ & $23.6 \pm 3.3$ & $23.6 \pm 3.8$ & $0.977^{\text {b }}$ \\
ASA classification & & & $0.371^{\text {a }}$ \\
$\quad$ Class I & $7 / 32(21.9 \%)$ & $6 / 21(28.6 \%)$ & \\
$\quad$ Class II & $25 / 32(78.1 \%)$ & $14 / 21(66.7 \%)$ & \\
Class III & $0 / 32(0.0 \%)$ & $1 / 21(4.8 \%)$ & \\
$\quad$ Class IV & $0 / 32(0.0 \%)$ & $0 / 21(0.0 \%)$ & \\
Stone laterality & & & $0.538^{\text {a }}$ \\
$\quad$ Right & $14 / 32(43.8 \%)$ & $11 / 21(52.4 \%)$ & \\
$\quad$ Left & $18 / 32(56.3 \%)$ & $10 / 21(47.6 \%)$ &
\end{tabular}

Stone location

\begin{tabular}{|c|c|c|c|}
\hline Upper & $0 / 32(0.0 \%)$ & $0 / 21(0.0 \%)$ & \\
\hline Middle & $1 / 32(3.1 \%)$ & 0/21 (0.0\%) & \\
\hline Lower & 2/32 (6.3\%) & $3 / 21(14.3 \%)$ & \\
\hline Pelvis & $3 / 32(9.4 \%)$ & $6 / 21(28.6 \%)$ & \\
\hline Multiple & 26/32 (81.3\%) & $12 / 21(57.1 \%)$ & \\
\hline Stone size $(\mathrm{cm})$ & $3.2 \pm 1.5$ & $3.3 \pm 1.6$ & $0.927^{d}$ \\
\hline Staghorn stone & 19/32 (59.4\%) & 9/21 (42.9\%) & $0.272^{a}$ \\
\hline Guy's stone score & & & $0.423^{\mathrm{a}}$ \\
\hline Grade I & $3 / 32(9.4 \%)$ & $5 / 21(23.8 \%)$ & \\
\hline Grade II & 10/32 (31.3\%) & $5 / 21(23.8 \%)$ & \\
\hline Grade III & 15/32 (46.9\%) & $10 / 21(47.6 \%)$ & \\
\hline Grade IV & $4 / 32(12.5 \%)$ & $1 / 21(4.8 \%)$ & \\
\hline Hounsfield unit & $976 \pm 265$ & $1103 \pm 369$ & $0.150^{b}$ \\
\hline Hydronephrosis & & & $0.615^{a}$ \\
\hline None & $8 / 32(25.0 \%)$ & $5 / 21(23.8 \%)$ & \\
\hline Mild & 23/32 (71.9\%) & 14/21 (66.7\%) & \\
\hline Severe & $1 / 32(3.1 \%)$ & $2 / 21$ (9.5\%) & \\
\hline Pre-operative PCN & & & $0.374^{c}$ \\
\hline Not done & $30 / 32(93.8 \%)$ & 18/21 (85.7\%) & \\
\hline Done & $2 / 32(6.3 \%)$ & $3 / 21(14.3 \%)$ & \\
\hline
\end{tabular}

$A E$ angioembolization, ASA American Society of Anesthesiologists, UPJ ureteropelvic junction, IPA infundibulopelvic angle, $P C N$ percutaneous nephrostomy

Results of continuous variables expressed as mean \pm standard deviation ${ }^{\text {a Chi-square }}$

bUnpaired t-test

'Fisher's exact test

${ }^{\mathrm{d}}$ Mann Whitney test significant factor predicting the occurrence of bleeding complications requiring angioembolization. El-Nahas et al. retrospectively analyzed 39 of 3878 PCNL procedures that required angioembolization [5]. Their multivariate analysis identified upper calyceal puncture, solitary kidney, staghorn stone, multiple punctures and inexperienced surgeon as significant risk factors. The identified factors from these studies however, did not differ from known factors for bleeding in general after PCNL from many other studies [12-15]. This shows that studies fail to identify factors that specifically cause severe bleeding requiring angioembolization after PCNL, partly because they compare the results between patients who underwent angioembolization and patients who did not have any bleeding complications.

On this basis, the current study was designed to analyze risk factors of angioembolization after PCNL by dividing the patients who received transfusion after PCNL into two groups: those who underwent angioembolization and those who did not. In our study, the usual factors known for bleeding after PCNL from many other studies such as stone complexity, degree of hydronephrosis, operation time, etc., did not show significant difference between the two groups, implying that these factors may be risk factors for bleeding in general after PCNL, but they are not the decisive factors causing severe bleeding requiring angioembolization. Therefore, to identify the critical factor, we attempted to determine whether the puncture itself was performed correctly by examining postoperative CT scans of patients who received transfusions after PCNL. This, to the best of our knowledge, was not done in previous studies.

Establishing a proper renal access is the most crucial step in the PCNL procedure, but it is a process that is difficult to master. A surgeon has to mentally visualize the three dimensional anatomy of the pelvocalyceal system from two dimensional images obtained with CT scans or fluoroscope. Ideally, the fornix or papilla of the posterior calyx should be punctured and the trajectory of the tract should be within 20 degrees posterior to the frontal plane of the kidney. In the current study, incorrect puncture was seen in 14/21 (66.7\%) patients who underwent angioembolization after PCNL, whereas it was seen in 11/21 (34.4\%) patients who did not undergo angioembolization, which showed statistical significance $(p=0.021)$. On multivariable regression analysis, puncture correctness was found to be the only significant factor with an OR of $3.818,95 \%$ CI of $1.192-12.231$ and $p$ value of 0.024 . This result suggests that adhering to the basics of renal puncture is paramount in preventing severe bleeding after PCNL. Not only will this decrease the risk of severe bleeding by traversing the avascular plane of Brödel's line, but it provides a relatively straight entry into the pelvis in the prone position [4]. If the fornix or 
Table 2 Comparison of procedural details between patients who did not undergo angioembolization and patients who underwent angioembolization after percutaneous nephrolithotomy

\begin{tabular}{|c|c|c|c|}
\hline & No AE & $\mathrm{AE}$ & $p$ value \\
\hline Operation time (minutes) & $114.0 \pm 55.8$ & $90.4 \pm 34.5$ & $0.187^{a}$ \\
\hline Location of access & & & $0.266^{\mathrm{b}}$ \\
\hline Lower pole & $20 / 32(62.5 \%)$ & $17 / 21(81.0 \%)$ & \\
\hline Other (middle or upper) & $11 / 32(34.4 \%)$ & $3 / 21(14.3 \%)$ & \\
\hline Lower pole \& other & $1 / 32(3.1 \%)$ & $1 / 21(4.8 \%)$ & \\
\hline Number of tracts & & & $1.000^{c}$ \\
\hline 1 & $31 / 32(96.9 \%)$ & 20/21 (95.2\%) & \\
\hline 2 & $1 / 32(3.1 \%)$ & $1 / 21(4.8 \%)$ & \\
\hline Puncture correctness & & & $0.021^{b}$ \\
\hline Correct & $21 / 32(65.6 \%)$ & $7 / 21(33.3 \%)$ & \\
\hline Incorrect & $11 / 32(34.4 \%)$ & $14 / 21(66.7 \%)$ & \\
\hline Stone free rate & $24 / 32(75.0 \%)$ & 15/21 (71.4\%) & $1.000^{\mathrm{b}}$ \\
\hline Interval between surgery and angioembolization (days) & NR & $7.4 \pm 6.4$ & \\
\hline Angiographic findings & NR & & \\
\hline Pseudoaneurysm & & 15/21 (71.4\%) & \\
\hline AVF & & $5 / 21(23.8 \%)$ & \\
\hline Both & & $1 / 21(4.8 \%)$ & \\
\hline
\end{tabular}

$A E$ angioembolization, UPJ ureteropelvic junction, AVF arteriovenous fistula, NR not relevant

Results of continuous variables expressed as mean \pm standard deviation

${ }^{a}$ Mann-Whitney U test

${ }^{\mathrm{b}} \mathrm{Chi}$-square test

'Fisher's exact test

papilla is missed and the infundibulum of the posterior calyx is punctured, injury to the interlobar arteries may occur [16]. Additionally, if the anterior calyx is punctured, the tract does not traverse Brödel's line, leading to an increased risk of severe bleeding [16]. There will also be an acute angle between the tract and renal pelvis, leading to more torque and an increased chance of bleeding.

Recently, there were reports that suggested nonpapillary or infundibular renal puncture was not associated with higher bleeding complications compared to papillary puncture [17]. However, these reports are from studies from single center and we feel that the evidence is not enough to suggest that non-calyceal or nonpapillary puncture is as safe as its counterpart. There is also the issue of infundibular stricture. If the puncture is not done through the calyx or papilla and done through the infundibulum, deep injury to the surrounding tissue may lead to infundibular stricture $[18,19]$.

Table 3 Multivariable logistic regression analysis to find out predictive factors of angioembolization after percutaneous nephrolithotomy in patients who received transfusion

\begin{tabular}{lllc}
\hline & OR & $95 \%$ Cl & $p$ value \\
\hline Puncture correctness & 3.818 & $1.192-12.231$ & 0.024 \\
\hline
\end{tabular}

$\mathrm{OR}$ odds ratio, $\mathrm{Cl}$ confidence interval, UPJ ureteropelvic junction
There were 7 patients who, despite their puncture being correct on postoperative CT scans, underwent angioembolization. It was noted that a high proportion of these patients, 5 of 7 (71.4\%), had a renal pelvis stone with extension into the upper ureter that was approached by the lower pole calyx. When selecting a pole for puncture, it is recommended that the pole that provides the most straight line along the stone axis be selected [20]. If this principle is not kept, the angle between the tract and the stone axis may become too acute, leading to excessive torque or a change in the direction of the tract, which can cause injury to the adjacent parenchyma with its vascular supply (Fig. 3) [20]. Some suggest that for best access to the ureteropelvic junction (UPJ), a pole whose calyx forms an angle of 90 degrees or more with the UPJ should be chosen [16]. When the angle was calculated for these 5 patients, it was 64.4 degrees, suggesting the possibility of excessive torque. If the middle or upper pole calyx was selected for renal puncture for these patients, bleeding could have been avoided. These results suggest that excessive torque may be one of the critical causes of severe bleeding leading to angioembolization after PCNL.

There were several limitations in the current study. First, the case number was relatively small, which was inevitable considering the very low incidence of severe 

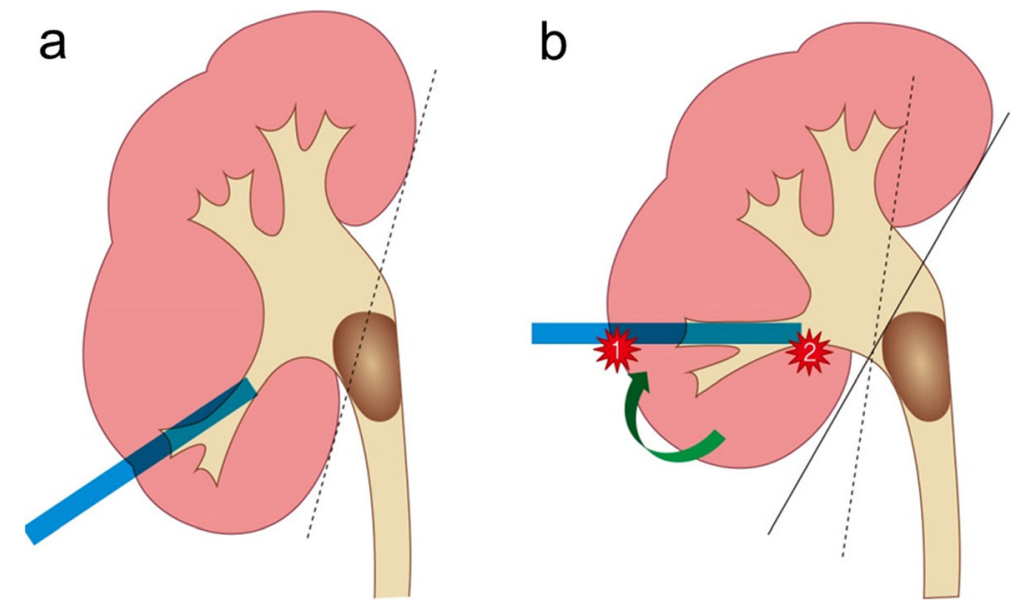

Fig. 3 Renal pelvis stone with upper ureter extension approached by a lower pole calyceal approach (a). To approach the stone, excessive torque may be necessary which may cause bleeding (b)

bleeding requiring angioembolization after PCNL. Second, there was the possibility of selection bias because the study was retrospective. CT scans were not routinely performed for patients who received blood transfusion after PCNL. Patients who did not undergo a postoperative CT scan were excluded because of the study purpose. Third, we performed all of our PCNLs with patients in the prone position with fluoroscopic assistance using a 30F Amplatz sheath. Therefore, the results would not be applicable to PCNLs performed in the supine position, or PCNLs using smaller caliber sheaths, such as mini-PCNLs or ultramini-PCNLs.

\section{Conclusions}

In the current study, we were able to identify whether the fornix or papilla of the posterior calyx was punctured and the trajectory of the tract was within Brödel's line by examining postoperative $\mathrm{CT}$ scans of patients who received transfusions after PCNL. When this principle of anatomically correct renal puncture was not followed, the risk of severe bleeding requiring angioembolization after PCNL significantly increased. Our results emphasize the importance of the basic principle of renal puncture for PCNL.

\section{Abbreviations}

AE: Angioembolization; ASA: American Society of Anesthesiologists; BMI: Body mass index; Cl: Confidence interval; CT: Computed tomography; OR: Odds ratio; PCN: Percutaneous nephrostomy; PCNL: Percutaneous nephrolithotomy; SFU: Society for Fetal Urology; UPJ: Ureteropelvic junction

\section{Acknowledgements}

Not applicable.

\section{Authors' contributions}

HYK contributed to project development, data collection, data analysis and manuscript writing/editing. KWL contributed to data collection. DSL contributed to project development, data collection, data analysis and manuscript writing/editing. All authors read and approved the final manuscript.

\section{Funding}

The authors declare that they received no funding for this study.

\section{Availability of data and materials}

The datasets used and/or analyzed during the current study are available from the corresponding author on reasonable request.

\section{Ethics approval and consent to participate}

The Institutional Review Board of the Catholic University of Korea, St. Vincent's Hospital approved the study protocol (approval number, VC19RESI0160).

\section{Consent for publication}

Not applicable.

\section{Competing interests}

The authors declare that they have no competing interests.

\section{Author details}

'Department of Urology, St. Vincent's Hospital, College of Medicine, The Catholic University of Korea, 93, Jungbu-daero, Paldal-gu, Suwon-si, Gyeonggi-do 16247, Republic of Korea. ${ }^{2}$ Department of Urology, Seoul St. Mary's Hospital, College of Medicine, The Catholic University of Korea, 222, Banpo-daero, Seocho-gu, Seoul 06591, Republic of Korea.

Received: 25 October 2019 Accepted: 2 March 2020 Published online: 11 March 2020

References

1. Schultheiss D, Engel RM, Crosby RW, Lees GP, Truss MC, Jonas U. Max Brodel (1870-1941) and medical illustration in urology. J Urol. 2000;164(4): $1137-42$.

2. Ko R, Soucy F, Denstedt JD, Razvi H. Percutaneous nephrolithotomy made easier: a practical guide, tips and tricks. BJU Int. 2008;101(5):535-9.

3. Miller NL, Matlaga BR, Lingeman JE. Techniques for fluoroscopic percutaneous renal access. J Urol. 2007:178(1):15-23.

4. Sampaio FJ, Zanier JF, Aragao AH, Favorito LA. Intrarenal access: 3dimensional anatomical study. J Urol. 1992;148(6):1769-73.

5. El-Nahas AR, Shokeir AA, El-Assmy AM, Mohsen T, Shoma AM, Eraky I, et al. Post-percutaneous nephrolithotomy extensive hemorrhage: a study of risk factors. J Urol. 2007;177(2):576-9. 
6. Srivastava A, Singh KJ, Suri A, Dubey D, Kumar A, Kapoor R, et al. Vascular complications after percutaneous nephrolithotomy: are there any predictive factors? Urology. 2005;66(1):38-40.

7. Thomas K, Smith NC, Hegarty N, Glass JM. The Guy's stone score--grading the complexity of percutaneous nephrolithotomy procedures. Urology. 2011;78(2):277-81.

8. Beetz R, Bokenkamp A, Brandis M, Hoyer P, John U, Kemper MJ, et al. Diagnosis of congenital dilatation of the urinary tract. Consensus Group of the Pediatric Nephrology Working Society in cooperation with the pediatric urology working Group of the German Society of urology and with the pediatric urology working Society in the Germany Society of pediatric surgery. Urologe A. 2001;40(6):495-507 quiz 508-499.

9. Turk C, Petrik A, Sarica K, Seitz C, Skolarikos A, Straub M, et al. EAU guidelines on interventional treatment for urolithiasis. Eur Urol. 2016;69(3): 475-82.

10. Fernstrom I, Johansson B. Percutaneous pyelolithotomy. A new extraction technique. Scand J Urol Nephrol. 1976;10(3):257-9.

11. Seitz C, Desai M, Hacker A, Hakenberg OW, Liatsikos E, Nagele U, et al. Incidence, prevention, and management of complications following percutaneous nephrolitholapaxy. Eur Urol. 2012;61(1):146-58.

12. Akman T, Binbay M, Sari E, Yuruk E, Tepeler A, Akcay M, et al. Factors affecting bleeding during percutaneous nephrolithotomy: single surgeon experience. J Endourol. 2011;25(2):327-33.

13. Keoghane SR, Cetti RJ, Rogers AE, Walmsley BH. Blood transfusion, embolisation and nephrectomy after percutaneous nephrolithotomy (PCNL). BJU Int. 2013;111(4):628-32.

14. Kukreja R, Desai M, Patel S, Bapat S, Desai M. Factors affecting blood loss during percutaneous nephrolithotomy: prospective study. J Endourol. 2004; 18(8):715-22.

15. Lee JK, Kim BS, Park YK. Predictive factors for bleeding during percutaneous nephrolithotomy. Korean J Urol. 2013;54(7):448-53.

16. Sampaio FJ. Renal anatomy. Endourologic considerations. Urol Clin North Am. 2000;27(4):585-607 vii.

17. Kallidonis P, Kyriazis I, Kotsiris D, Koutava A, Kamal W, Liatsikos E. Papillary vs nonpapillary puncture in percutaneous Nephrolithotomy: a prospective randomized trial. J Endourol. 2017;31(S1):S4-9.

18. Parsons JK, Jarrett TW, Lancini V, Kavoussi LR. Infundibular stenosis after percutaneous nephrolithotomy. J Urol. 2002;167(1):35-8.

19. Weir MJ, Honey JD. Complete infundibular obliteration following percutaneous nephrolithotomy. J Urol. 1999;161(4):1274-5.

20. Sharma GR, Maheshwari PN, Sharma AG, Maheshwari RP, Heda RS, Maheshwari SP. Fluoroscopy guided percutaneous renal access in prone position. World J Clin Cases. 2015;3(3):245-64.

\section{Publisher's Note}

Springer Nature remains neutral with regard to jurisdictional claims in published maps and institutional affiliations.

Ready to submit your research? Choose BMC and benefit from:

- fast, convenient online submission

- thorough peer review by experienced researchers in your field

- rapid publication on acceptance

- support for research data, including large and complex data types

- gold Open Access which fosters wider collaboration and increased citations

- maximum visibility for your research: over $100 \mathrm{M}$ website views per year

At $\mathrm{BMC}$, research is always in progress.

Learn more biomedcentral.com/submissions 